\title{
Special Issue on Deep Structured Learning for Natural Language Processing
}

The landscape of deep structured learning has changed in recent years. Many new trends and techniques have entered the arena. This editorial on "Deep Structured Learning for Natural Language Processing" brings out the innovative applications of deep structured learning for various dimensions of natural language processing (NLP). To this point, this editorial gives the researcher and practitioner's orientation on recent developments in NLP and provides an opportunity for relevant and qualitative research.

This special section provides research articles that were selected following a rigorous review process focusing on various applications of deep learning for NLP. Overall, the special section contains 5 articles. Specifically, the first article. From a practical perspective, today's crime rate increases exponentially, leading to numerous serious threats in society. Due to this research, interest in cyberbullying has acquired increased research attention in recent days. Forefronts in machine learning and NLP are the two most important cutting-edge techniques that reduce the risk of cyberbullying in contemporary modern society. A new detection algorithm based on fast text detection and word similarity is introduced. It further analyzes the cyberbullying characteristics from the lexical as well as the syntactic viewpoints. From the experimental observation, it is envisioned that detection accuracy and recall measures are improved compared to the existing schemes.

A scheme called "deep interactive memory network for aspect-level sentiment analysis" is explored to effectively study the subjective information. It makes use of deep learning techniques to extract opinions from the massively large datasets. Imbalanced Twitter sentiment analysis is the systematic issue deals with class imbalances and its effects. A research article is proposed to analyze the emotions in a big corpus of imbalanced tweets using deep learning techniques. Another important research area is big data analytics and NLP applications. Against this background, research articles emphasis on transnational happiness study on big data using artificial intelligence and deep learning techniques. Next, knowledge discovery plays a vital role when dealing with big data. Effective ways of retrieving knowledge from news texts are analyzed in depth using NLP and deep learning methods. Finally, citation recommendation in massively generated big data is often a crucial task. This process is made easier with model-enhanced graph modeling techniques. This approach reduces the complexity rate and assists in effective citation process.

This special issue emphasis on various aspects NLP using deep learning techniques. The articles published in this special section contribute to the application of NLP in various domains, which includes NLP and deep learning for big data analytics, recommendation, and linguistic analysis. We thank the authors for submitting good quality research contributions reflecting the theme of

\footnotetext{
ACM Reference format:

Dr. Gunasekaran Manogaran, Dr. Hassan Qudrat-Ullah, and Dr. Qin Xin. 2021. Special Issue on Deep Structured Learning for Natural Language Processing. ACM Trans. Asian Low-Resour. Lang. Inf. Process. 20, 1, Article 1 (March 2021), 2 pages. https://doi.org/10.1145/3436206
}

(c) 2021 Copyright held by the owner/author(s).

2375-4699/2021/03-ART1

https://doi.org/10.1145/3436206

ACM Trans. Asian Low-Resour. Lang. Inf. Process., Vol. 20, No. 1, Article 1. Publication date: March 2021. 
the special issue. Also, a special thanks to the reviewers for taking their time and efforts to review the manuscript qualitatively.

Dr. Gunasekaran Manogaran, Big Data Scientist, University of California, Davis, USA gmanogaran@ucdavis.edu https://scholar.google.com/citations?user=hO2LWCIAAAAJ\&hl=en http://ucdavis.academia.edu/GunasekaranManogaran https://jmie.pure.elsevier.com/en/persons/gunasekaran-manogaran

Dr. Hassan Qudrat-Ullah, Professor of Decision Sciences, School of Administrative Studies, York University, Toronto, Canada hassanq@yorku.ca https://www.researchgate.net/profile/Hassan_Qudrat-Ullah https://scholar.google.com/citations?user=Ua7vaHQAAAAJ\&hl=en

Dr. Qin Xin, Full Professor of Computer Science, Faculty of Science and Technology, University of the Faroe Islands, Faroe Islands. qinx@setur.fo https://www.researchgate.net/profile/Qin_Xin3 https://scholar.google.co.in/citations?user=ox7IO1YAAAAJ\&hl=en Guest Editors 